\title{
Editorial Response to Hepato-Biliary Disease Resection for Patients with Advanced Epithelial Ovarian Cancer: Prognostic Role and Optimal Cytoreduction
}

\author{
Sarah S. Lee, MD, MBA, and Bhavana Pothuri, MD, MS \\ Department of Obstetrics and Gynecology, Division of Gynecologic Oncology, Perlmutter Cancer Center, New York \\ University Grossman School of Medicine, New York, NY
}

In this study by Di Donato et al., ${ }^{1}$ complete cytoreduction was achieved in $71.6 \%$ of the patients who had advanced epithelial ovarian cancer with hepatobiliary spread. However, the patients with hepatobiliary spread were less likely than the overall population to achieve complete cytoreduction. For $42 \%$ of the patients, the reason for incomplete cytoreduction was not associated with disease in the hepatobiliary area. The overall complication rate was $58.2 \%$, and the severe complication rate was only $10.5 \%$ for the patients who underwent hepatobilary resection as part of their debulking surgery.

Disease involving the porta hepatis was previously thought to be a contraindication to surgical cytoreduction. ${ }^{2,3}$ Disease in the hepatobiliary area may be related to an overall increased disease burden or a marker of worse disease, and may be associated with unresectable disease.

In this study, the majority of the patients with spread to the hepatobiliary area had liver parenchymal involvement, and 6 of the 19 patients who did not achieve complete resection had involvement of the porta hepatis. The patients with hepatobiliary involvement who achieved complete cytoreduction had improved progression-free survival (PFS) and overall survival (OS).

Although there were no differences between the patients with and without hepatobiliary involvement who achieved complete cytoreduction, those with hepatobiliary disease

(C) Society of Surgical Oncology 2020

First Received: 31 July 2020

Accepted: 9 August 2020;

Published Online: 18 August 2020

B. Pothuri, MD, MS

e-mail: Bhavana.pothuri@nyulangone.org did have a shorter median PFS, suggesting that hepatobiliary spread may be associated with worse outcomes. However, the benefit of surgical resection, in terms of no gross residual disease, was associated with improvement in median PFS and OS, highlighting the importance of surgical cytoreduction for these patients. Hepatobiliary disease, and specifically porta hepatis disease, should not automatically be considered a contraindication to debulking surgery in ovarian cancer. A model of laparoscopic investigation for determining surgical resectability includes superficial liver metastasis, intraparenchymal liver disease, or both, but does not address porta hepatis disease. ${ }^{4}$ The rate for resectability of the hepatobiliary area can be dependent on the surgical expertise and experience of the surgeon. ${ }^{5}$ Much of optimal surgical debulking can require an interdisciplinary approach requiring the availability of multiple subspecialties. ${ }^{6}$

A remaining question to be answered is the outcome for patients with hepatobiliary disease who were counseled to undergo neoadjuvant chemotherapy instead of primary cytoreduction. Because neoadjuvant therapy aims to increase the likelihood of resectable disease at the time of interval debulking, patients with imaging concerns about hepatic involvement may be likely to undergo neoadjuvant chemotherapy. It would be interesting to determine whether patient counseling differed depending on the location of the hepatobiliary spread (i.e., porta-hepatis disease or multiple liver parenchymal metastasis) and also whether PFS or OS differed for these patients based on the types of initial management, allowing the optimal strategy for them to be better defined.

Finally, the patients included in this study had a median age of 54 years and a body mass index of 24, which may have contributed to selection bias for a younger and 
healthier patient population than the average population of patients with advanced ovarian cancer. Perhaps they contributed to the low rate of severe complications for this population of patients undergoing surgery. Patient selection for this highly complex surgery is key to successful and improved outcomes, as described by the authors.

Overall, this report adds to the literature on hepatobiliary disease resection showing a high rate of gross resection for this population of patients with advanced ovarian cancer. Although hepatobiliary disease should not be an absolute contraindication to debulking surgery, careful patient selection with specialized surgical teams is important to the achievement of optimal outcomes. Additional information on the surgical techniques used and the patient selection factors that may have contributed to the high rate of resectability would be beneficial. Confirmation of these findings in a prospective multi-center trial would be valuable in the decision-making for these patients.

DISCLOSURES There are no conflict of interest.

\section{REFERENCES}

1. Di Donato V, Giannini A, D’Oria O, Schiavi MC, Di Pinto A, Fischetti M, et al. Hepato-biliary disease resection in patients with advanced epithelial ovarian cancer: prognostic role and optimal cytoreduction. Ann Surg Oncol. 2020. https://doi.org/10.1245/s10 434-020-08989-3.

2. Vergote I, du Bois A, Amant F, Heitz F, Leunen K, Harter P. Neoadjuvant chemotherapy in advanced ovarian cancer: on what do we agree and disagree? Gynecol Oncol. 2013;128:6-11.

3. Suidan RS, Ramirez PT, Sarasohn DM, Teitcher JB, Iyer RB, Zhou Q, et al. A multicenter assessment of the ability of preoperative computed tomography scan and CA-125 to predict gross residual disease at primary debulking for advanced epithelial ovarian cancer. Gynecol Oncol. 2017;145:27-31.

4. Fagotti A, Ferrandina G, Fanfani F, Garganese G, Vizzielli G, Carone V, et al. Prospective validation of a laparoscopic predictive model for optimal cytoreduction in advanced ovarian carcinoma. Am J Obstet Gynecol. 2008;199:642 e1-6.

5. Zivanovic O, Eisenhauer EL, Zhou Q, Iasonos A, Sabbatini P, Sonoda Y, et al. The impact of bulky upper abdominal disease cephalad to the greater omentum on surgical outcome for stage IIIC epithelial ovarian, fallopian tube, and primary peritoneal cancer. Gynecol Oncol. 2008;108:287-92.

6. Wang M, Zhou J, Zhang L, Zhao Y, Zhang N, Wang L, et al. Surgical treatment of ovarian cancer liver metastasis. Hepatobil Surg Nutr. 2019;8:129-37.

Publisher's Note Springer Nature remains neutral with regard to jurisdictional claims in published maps and institutional affiliations. 